\title{
Simulation of Novel NEMS Contact Switch Using MRTD with Alterable Steps
}

\author{
Wen-Ge Yu, ${ }^{1}$ Kang-Qu Zhou, ${ }^{2}$ Zheng-Zhong Wu, ${ }^{1}$ Ting-Hong Yang, ${ }^{1}$ and Jing Zhao ${ }^{1}$ \\ ${ }^{1}$ Department of Mathematics, Logistic Engineering University, Chongqing 400016, China \\ ${ }^{2}$ Department of Industrial Engineering, Chongqing University of Technology, Chongqing 400050, China
}

Correspondence should be addressed to Wen-Ge Yu, yuwenge@126.com

Received 22 September 2009; Revised 12 January 2010; Accepted 28 February 2010

Academic Editor: Felix A. Buot

Copyright (C) 2010 Wen-Ge Yu et al. This is an open access article distributed under the Creative Commons Attribution License, which permits unrestricted use, distribution, and reproduction in any medium, provided the original work is properly cited.

\begin{abstract}
In order to apply Radio Frequency Micro-nano-Electro-Mechanical System (MEMS/NEMS) technologies to produce miniature, high isolation, low insertion loss, good linear characteristic, and low power consumption microwave switches, we present a novel NEMS switch with nanoscaling in this paper through the analysis of electrics and mechanics of the RF switch. The measured data show the pull-in voltage of $24.1 \mathrm{~V}$ and the good RF performance of the insertion loss of below $-10 \mathrm{~dB}$ at $0 \mathrm{GHz}$ on the "on" state, and the isolation of beyond $-40 \mathrm{~dB}$ at $0-40 \mathrm{GHz}$ on the "off" state, indicating that the witch is suitable for the $0-40 \mathrm{GHz}$ applications. Our analysis shows that the NEMS switch not only can work in wide frequency bands, but also has better isolation performance in lower frequency, thus extending the application to the lower band. The Haar-wavelet-based multiresolution time domain (MRTD) with compactly supported scaling function is used for modeling and analyzing the nanomachine switch for the first time. The major advantage of the MRTD algorithms is their capability to develop real-time time and space adaptive grids through the efficient thresholding of the wavelet coefficients. The error between the measured and computed results is below $5 \%$, this indicated that the Haar-wavelet-based multiresolution time domain was suitable for simulating the nano-scaling contact switch.
\end{abstract}

\section{Introduction}

As the key device of radio frequency or high frequency transmission systems, RF switch is widely used in civil and military applications of RF, microwave, and millimeterwave circuits and systems. Previously, RF switching is implemented by using p-i-n diodes and GaAs MESFETs in the form of junction field-effect transistor- (JFET-)based semiconductor switches [1-4]. Recently, Radio frequency nano-electro-mechanical system (RF-NEMS) switches have attracted increasing attention over the traditional ones, due to their low insertion loss, high isolation, and low power consumption.

NEMS switches are mainly categorized as contact and capacitive switches. In contrast with the capacitive one, such as the switch reported by $[5,6]$, the contact switch has a wider band down to the DC frequency. However, the cantilever structure of the contact switch is too sensitive to the stress of the dielectrics, adding to the fabrication difficulties [7, 8]. Researchers in the University of Michigan developed an all-metal broadside-series switch [5]. But this structure cannot avoid RF signal isolation, which results in rapidly degrading performance on high frequency band. Another dielectric membrane broadside-series switch has solved the problem of signal isolation. Yet the upper electrodes and contact metal are on top of and beneath the membrane, respectively, which increase the fabrication complexities.

In this paper, we present a novel NEMS switch, including contact metal bar and upper electrodes both underneath the $\mathrm{SiON}$ bridge. It can solve all the problems described above, such as isolation of the RF signal from bias, insensitivity to the membrane stress, and simplification of the fabrication process. Additionally, the closer distance between the upper and lower electrodes reduces the pull-in voltage. According to the measured data, the designed and fabricated switch exhibits low insertion loss, high isolation, and low pull-in voltage. 


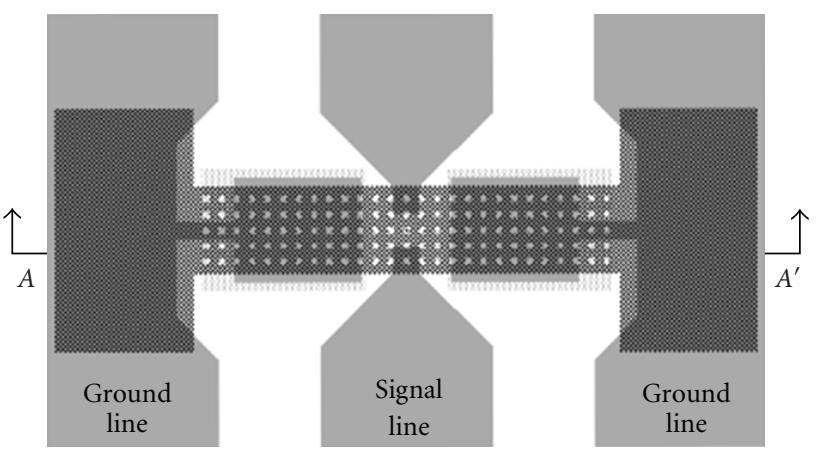

(a) Top view of the switch
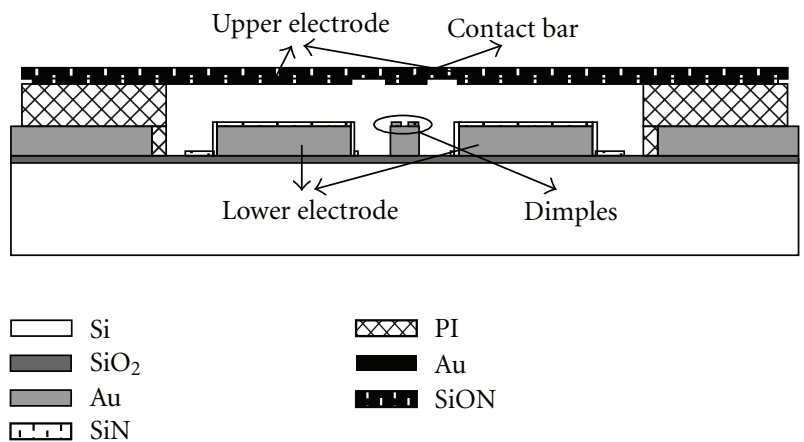

(b) Cross view of the switch along $A-A^{\prime}$ derection

FIgURE 1: Main structure of the switch.

The finite-difference time-domain (FDTD) method is widely used for solving problems related to electromagnetism. However, there still exist many restrictive factors, such as memory shortage, CPU time, and memory needs. We first adopted the method of the Haar-wavelet-based multiresolution time domain (MRTD) $[9,10]$ with compactly supported scaling function for a full three-dimensional (3D) wave to Yee's staggered cell to analyze and simulate the NEMS switch. The major advantage of the MRTD algorithms is their capability to develop real-time and space adaptive grids through the efficient thresholding of the wavelet coefficients. By this technique, space discretization with only a few cells per wavelength gives relatively accurate results, leading to the reduction of both memory requirement and computation time. Associated with practical model, an uniaxial perfectly matched layer (UPML) absorbing boundary conditions [11] was then developed, and a three-dimensional formulation of the discrete difference equations arising from the Maxwell's system is extended to an inhomogeneous medium and applied to the analysis of the NEMS switch.

\section{Structure of the NEMS Switch}

The main structure of the designed switch is shown in Figure 1. The whole switch is fabricated on the silicon substrate with a silicon oxide layer on top of it. The central part of the signal line is separated to make a gap and is narrowed to get better isolation performance on the "off" state. An SiON bridge of $10000 \mathrm{~nm}$ long, $200 \mathrm{~nm}$ wide and
$10 \mathrm{~nm}$ thick is suspended across the gap and is fixed over the ground lines via a polyimide layer on the both sides. Arrays of holes, as shown in Figure 1(a), a gold bar clings to the bottom surface of the bridge center, making two overlapped areas together with the separated signal line. Several dimples are fabricated on the overlapped areas of the signal line, to make a good metal-metal contact between the signal line and the gold bar, as shown in Figure 1(b). In particular, the two side upper electrodes also cling to the bottom surface of the bridge and are positioned on the same plane of the contact bar. The RF signal can be isolated due to the separation of the bar and the upper electrodes, and the fabrication process can be simplified due to the same plane of them. The two side lower electrodes are connected to the ground lines, which are DC voltage insulated by the dielectric polyimide layer.

When the DC voltage is applied between the upper and lower electrodes, the bridge is pulled down toward the substrate to obtain a metal-metal contact between the contact bar and the signal line, resulting in the switch "on" state; otherwise, the switch keeps the "off" state.

\section{3D MRTD Algorithm}

3.1. Numerical Formulations of the 3D MRTD Method. Maxwell's curl equations in an isotropic medium are

$$
\begin{aligned}
& \nabla \times \vec{E}=-\mu \frac{\partial \vec{H}}{\partial t}, \\
& \nabla \times \vec{H}=\varepsilon \frac{\partial \vec{E}}{\partial t}+\sigma \vec{E},
\end{aligned}
$$

where $\varepsilon$ is permittivity, $\mu$ is permeability, $\sigma$ is electric conductivity. Each field component is expanded into scaling functions

$$
\phi_{u}(s)=\phi\left(\frac{s}{\Delta s-u}\right)
$$

and wavelets

$$
\psi_{u}(s)=\psi\left(\frac{s}{\Delta s-u}\right)
$$

where

$$
\phi(s)= \begin{cases}1, & s \in(0,1), \\ 0, & \text { otherelse }\end{cases}
$$

and $\psi(s)=\phi(2 s)-\phi(2 s-1)$.

Expansion and testing is performed for each spatial coordinate $s=\{x, y, z\}$ with corresponding discretization indices $u=\{k, l, m\}$, as well as for time with rectangular pulse $h_{n}(t)$. In compact notations, the $x$-directed electric field component in the staggered Yee's grid of size $\Delta x, \Delta y, \Delta z$ is represented as

$$
\begin{aligned}
& E_{x}(x, y, z, t) \\
& \quad=\sum_{k l m} \sum_{\xi \eta \zeta} \sum_{n}{ }_{n}^{x} E_{k+1 / 2, l, m}^{\xi \eta \zeta} \cdot \xi_{k+1 / 2}(x) \eta_{l}(y) \zeta_{m}(z) h_{n}(t),
\end{aligned}
$$

where $x=k \Delta x, y=l \Delta y, z=m \Delta z, t=n \Delta t$. 
The summation over $\xi \eta \zeta$ includes eight terms stemming from all the permutations of scaling functions and wavelets

$$
\xi \eta \zeta=\{\phi \phi \phi, \phi \phi \psi, \phi \psi \phi, \phi \psi \psi, \psi \phi \phi, \psi \phi \psi, \psi \psi \phi, \psi \psi \psi\} .
$$

The representation of the other field components is easily derived through permutation of the indices and follows the same rule as for standard FDTD scheme. Inserting the above expressions into the difference equations and performing a Galerkin test procedure leads to the following expressions for the electric field within each cell $\{k, l, m\}$ :

$$
\begin{aligned}
{ }_{3}^{x} E_{100}^{\xi \eta \zeta}- & { }_{0}^{x} E_{100}^{\xi \eta \zeta} \\
= & \frac{\Delta t}{\varepsilon} \\
& \cdot\left\{\frac{{ }_{1}^{z} H_{110}^{\xi \eta \zeta}-{ }_{1}^{z} H_{120}^{\xi \eta \zeta}}{\Delta y}-\frac{{ }_{1}^{y} H_{101}^{\xi \eta \zeta}-{ }_{1}^{y} H_{102}^{\xi \eta \zeta}}{\Delta z}-\sigma \cdot{ }_{1}^{x} E_{100}^{\xi \eta \zeta}\right\},
\end{aligned}
$$

where $\{0,1,2,3\}$ denotes $\{u, u+1 / 2, u-1 / 2, u+1\}$ for each $u=\{k, l, m, n\}$, respectively. In formula (7), there are three different $E_{x}$ values within one time step, being inconvenient for program design. In order to avoid the shortcoming, we adopt approximation as follows:

$$
{ }_{1}^{x} E_{100}^{\xi \eta \zeta}=\frac{1}{2}\left({ }_{3}^{x} E_{100}^{\xi \eta \zeta}+{ }_{1}^{x} E_{100}^{\xi \eta \zeta \zeta}\right) .
$$

Similar expressions are obtained for the other field components.

3.2. Absorbing Boundary Condition. The field computation domain must be limited in size because the computer cannot store unlimited amount of data. The computation domain must be large enough to enclose the structure of interest. In this paper, we adopted uniaxial perfectly matched layer (UPML) absorbing boundary conditions. Consider one dimension wave equation propagated along $+z$ direction:

$$
\left(\frac{\partial}{\partial z}-\frac{1}{v} \frac{\partial}{\partial t}\right) E_{x}-\frac{\sigma^{\prime}}{v} E_{x}=0
$$

where $\sigma^{\prime}=\sigma / \varepsilon, v$ is the phase velocity in the concerned volume. Since the conductivity $\sigma$ is projected in computation domain, it will result in numeric dispersion if we use directly discrete approximation for formula (9). Let $E_{x}(z, t)=$ $\widetilde{E}_{x}(z, t) e^{-\sigma^{\prime} t}$, then

$$
\left(\frac{\partial}{\partial z}-\frac{1}{v} \frac{\partial}{\partial t}\right) \widetilde{E}_{x}=0
$$

Its finite difference form is

$$
E_{x}^{k+1}(n)=E_{x}^{k-1}(n)+\frac{2 v \Delta t}{\Delta z} \sum_{i} b(i) E_{x}^{k}(n+i) .
$$

The difference form of formula (9) is

$$
E_{x}^{k+1}(n)=e^{-2 \sigma^{\prime} \Delta t} E_{x}^{k-1}(n)+\frac{2 v \Delta t}{\Delta z} e^{-\sigma^{\prime} \Delta t} \sum_{i} b(i) E_{x}^{k}(n+i),
$$

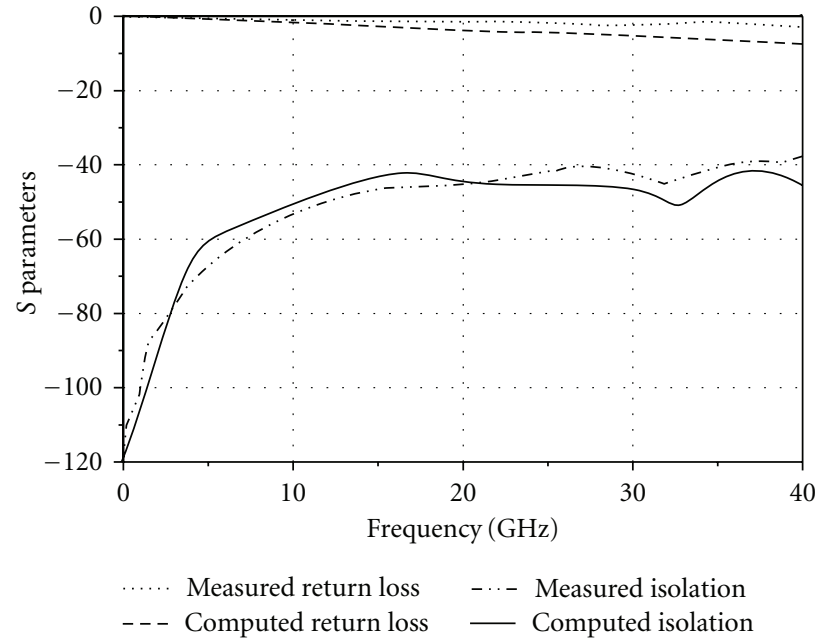

FIgURE 2: Comparison of the NEMS switch on the "off" state.

where $b(i)=\int(\partial \psi(x) / \partial x) \psi(x+i-1 / 2) d x$ are the MRTD coefficients.

The UPML material parameters are chosen to be $\sigma_{\xi(\xi=x, y, z)}=0$ for the inner computation region, while the maximum value of $\sigma$ at the end of the UPML region is chosen to be $\sigma_{\max }=1 /\left(150 \pi \Delta \sqrt{\varepsilon_{r}}\right)$, where $\Delta$ is the cell dimension perpendicular to the UPML interface to the regular region. The UPML region is backed by a perfect electric conductor wall implemented by applying the mirror principle.

\section{Calculated Results}

In microwave circuit analysis, Gauss impulse is generally selected as an excitation for smoothness in time domain and easy spectrum width setting. The width of gauss pulse is $T=$ 15 picoseconds. Assuming that the time delay is $t_{0}=3 T=$ 45 picoseconds, the response value of the frequency domain can be calculated by Fourier transforming the time domain value. In the course of computing, because of nano-scaling structure of the switch, we adopted double computation domain by the characteristic of MRTD algorithm's adaptive grids.

The $S$ parameters of the switch computed and measured for the switch "off" and "on" state are shown in Figure 2 and Figure 3, respectively. The computed curves based computation domain $50 \times 50 \times 50$ and $100 \times 100 \times 50$, space steps $\Delta x=\Delta y=0.02 \mathrm{~nm}, \Delta z=0.015 \mathrm{~nm}$ and $\Delta x=\Delta y=0.1 \mathrm{~nm}, \Delta z=0.015 \mathrm{~nm}$, respectively. From Figures 2 and 3, we can find that the computed results by MRTD method are in agreement with the measured results on the whole. The drifts between the measured value and the computed value by using MRTD are below $5 \%$, respectively. The characteristic parameters such as effective dielectric constant and the characteristic impedance in spectrum domain could be worked out by Fourier transition. 


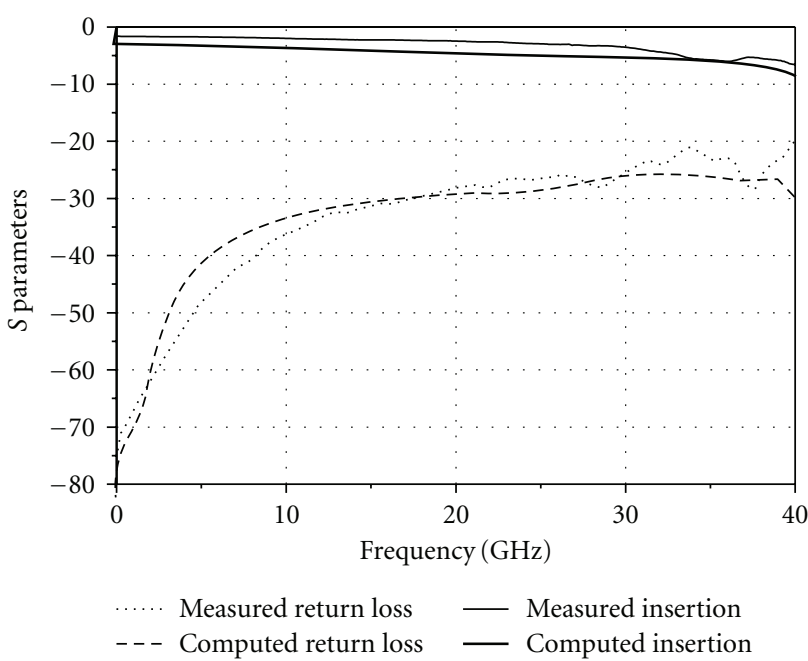

FIGURE 3: Comparison of the NEMS switch on the "on" state.

\section{Conclusion}

A novel NEMS switch has been presented in this paper; it performs excellently especially in low insertion loss, high isolation, and low power consumption. The measured and the computed results show the contact resistance of $0.8-$ $1.4 \Omega$, the pull-in voltage of $24.1 \mathrm{~V}$, the isolation of beyond $-40 \mathrm{~dB}$ at $0-40 \mathrm{GHz}$ on the "off" state, and the insertion loss of below $-10 \mathrm{~dB}$ at $0-40 \mathrm{GHz}$ on the "on" state. It is suitable for the $0-40 \mathrm{GHz}$ frequency band applications. MRTD method was used to model the structure of the switch. The algorithm is real-time and space adaptive grids through the efficient thresholding of the wavelet coefficients. Based on the characteristic of MRTD algorithm's adaptive grids, we adopted double computation domain, leading to a reduction of both memory requirement and computation time, but also we can find that the computed results by MRTD method are in agreement with the measured results on the whole. The error between the measured value and the computed value by using MRTD is in 5\%.

\section{Acknowledgments}

This work is supported by the National Natural Science Foundation of China (Project no. 60576048 10971227) and the Science Innovation Foundation of Logistical Engineering University, Chongqing, China (Project no. YZ07-41101).

\section{References}

[1] L. W. Ke, Y. J. Chan, and Y. C. Chiang, "Monolithic microwave AlGaAs/InGaAs doped-channel FET switches," Microwave and Optical Technology Letters, vol. 13, no. 1, pp. 47-49, 1996.

[2] K. Kawakyu, Y. Ikeda, M. Nagaoka, et al., "A novel resonanttype GaAs SPDT switchic with low distortion characteristics for $1.9 \mathrm{GHz}$ personal handy-phone system," in Proceedings of the IEEE MTT-S International Microwave Symposium Digest, vol. 2, pp. 647-650, San Franscisco, Calif, USA, June 1996.
[3] J. B. Muldavin and G. M. Rebeiz, "Inline capacitive and DCcontact MEMS shunt switches," IEEE Microwave and Wireless Components Letters, vol. 11, no. 8, pp. 334-336, 2001.

[4] R. Chan, R. Lesnick, D. Becher, and M. Feng, "Low-actuation voltage RF MEMS shunt switch with cold switching lifetime of seven billion cycles," Journal of Microelectromechanical Systems, vol. 12, no. 5, pp. 713-719, 2003.

[5] J. B. Muldavin and G. M. Rebeiz, "All-metal high-isolation series and series/shunt MEMS switches," IEEE Microwave and Wireless Components Letters, vol. 11, no. 9, pp. 373-375, 2001.

[6] G. M. Rebeiz and J. B. Muldavin, "RF MEMS switches and switch circuits," IEEE Microwave Magazine, vol. 2, no. 4, pp. 59-71, 2001.

[7] X.-F. Lei, Z.-W. Liu, Y. Xuan, L. Liu, Z. Li, and J. Liu, "Fabrication of a novel capacitive RF-MEMS switch," in Proceedings of Pacific Rim Workshop on Transducers and Micro/Nano Technologies, vol. 1, pp. 789-792, July 2002.

[8] D. Peroulis, S. Pacheco, K. Sarabandi, and L. P. B. Katehi, "MEMS devices for high isolation switching and tunable filtering," in Proceedings of the IEEE MTT-S International Microwave Symposium Digest, vol. 2, pp. 1217-1220, Boston, Mass, USA, June 2000.

[9] W. Yu, "Algorithm of four-resonances MEMS capacitive switch using ADI-FDTD," in Proceedings of the 7th World Congress on Intelligent Control and Automation (WCICA '08), pp. 42954298, Chongqing, China, June 2008.

[10] W. G. Yu, K. Q. Zhou, and T. H. Yang, "Modeling of novel modulated Helix induction MEMS switch on time-domain," Journal of Physics: Conference Series, vol. 48, no. 1, pp. 298302, 2006.

[11] G. Mur, "Absorbing boundary conditions for the finite-difference approximation of the time-domain electromagnetic-field equations," IEEE Transactions on Electromagnetic Compatibility, vol. 23, no. 4, pp. 377-382, 1981. 

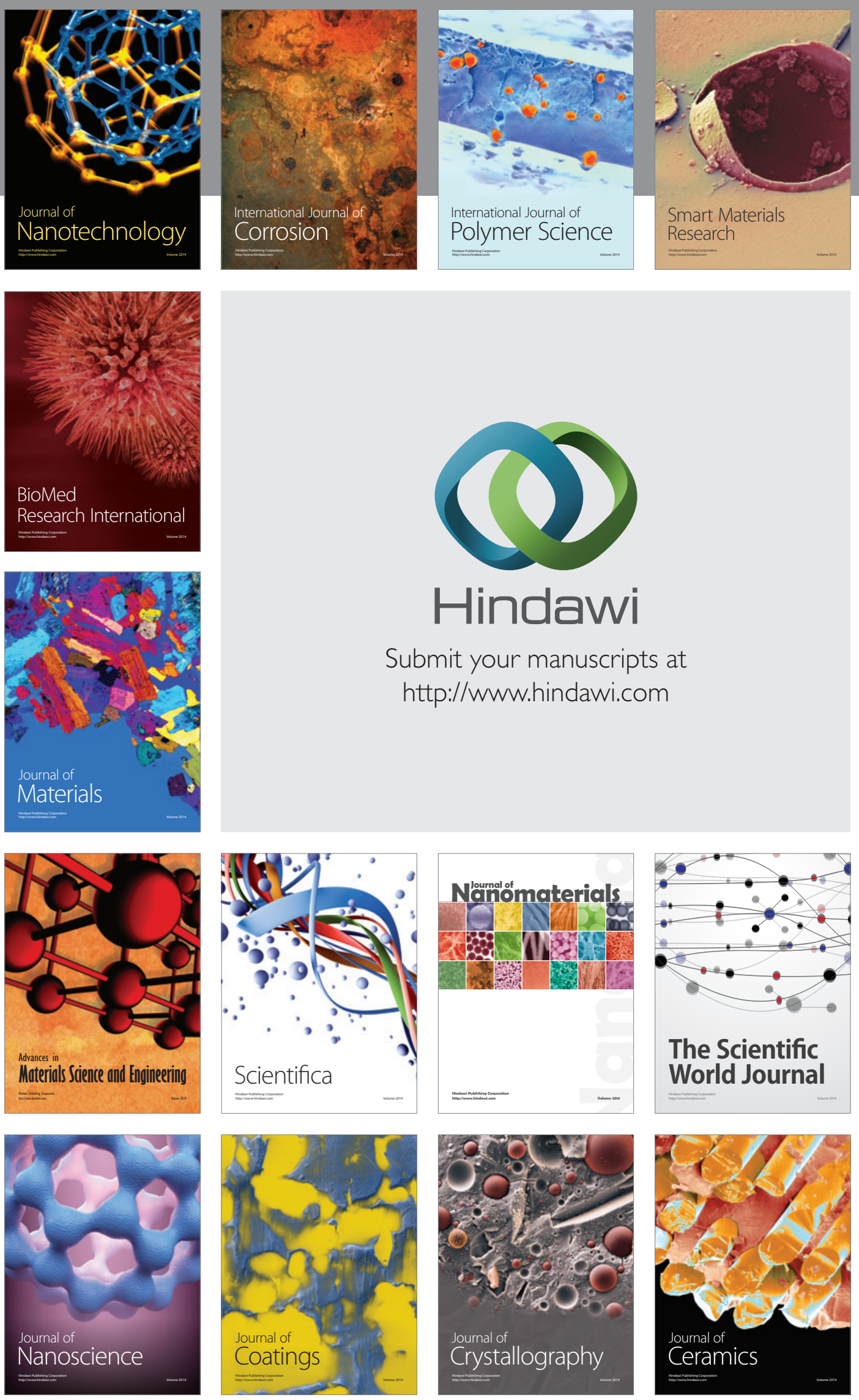

The Scientific World Journal

Submit your manuscripts at

http://www.hindawi.com

\section{World Journal}

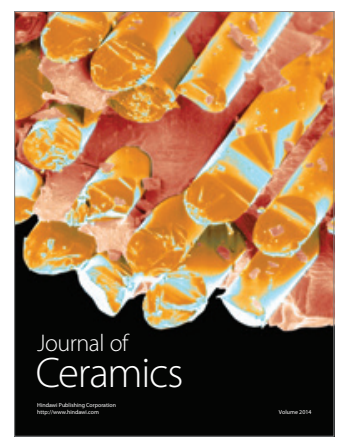

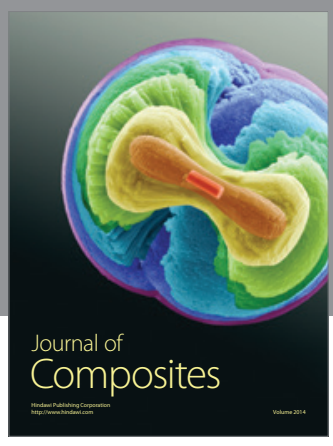
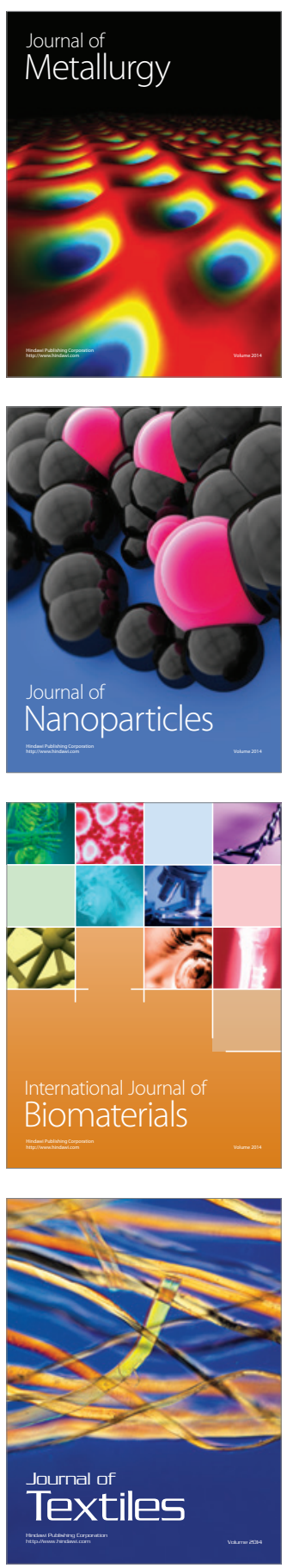